\title{
ABELIAN SUBALGEBRAS AND IDEALS OF MAXIMAL DIMENSION IN SUPERSOLVABLE AND NILPOTENT LIE ALGEBRAS
}

\author{
DAVID A. TOWERS
}

\author{
Department of Mathematics, Lancaster University \\ Lancaster LA1 4YF, England \\ d.towers@lancaster.ac.uk
}

\begin{abstract}
In this paper, we continue the study of abelian subalgebras and ideals of maximal dimension for finite-dimensional supersolvable and nilpotent Lie algebras. We show that supersolvable Lie algebras with an abelian subalgebra of codimension 3 contain an abelian ideal with the same dimension, provided that the characteristic of the underlying field is not two, and that the same is true for nilpotent Lie algebras with an abelian subalgebra of codimension 4 , provided that the characteristic of the field is greater than five.
\end{abstract}

Mathematics Subject Classification 2020: 17B05, 17B20, 17B30, 17B50.

Key Words and Phrases: Lie algebras, abelian subalgebra, abelian ideal, solvable, supersolvable, nilpotent.

\section{Introduction}

Throughout, $L$ will denote a Lie algebra of finite dimension $n$ over a field $F$. Denote by $\alpha(L)$ the maximal dimension of an abelian subalgebra of $L$, and by $\beta(L)$ the maximal dimension of an abelian ideal of $L$. These invariants have been the subject in much interest in many areas. For example, they are very useful invariants in the study of Lie algebra contractions and degenerations. 
There is a large literature, in particular for low-dimensional Lie algebras, see $[7,2,14,17,6]$, and the references given therein.

The first author dealing with the invariant $\alpha(\mathfrak{g})$ was Schur [16], who studied in 1905 the abelian subalgebras of maximal dimension contained in the Lie algebra of $n \times n$ square matrices. Schur proved that the maximum number of linearly independent commuting $n \times n$ matrices over a algebraically closed field is $\left[\frac{n^{2}}{4}\right]+1$, which is the maximal dimension of abelian ideals of Borel subalgebras in the general linear Lie algebra $\mathfrak{g l}(n)$ ( where $[x]$ denotes the integer part of a real number $x$ ). Initially, this result was obtained only over an algebraically closed field such as the complex number field. Almost forty years later, in 1944, Jacobson [8] gave a simpler proof of Schur's results, extending them from algebraically closed fields to arbitrary fields. This fact allowed several authors to gain insight into the abelian subalgebras of maximal dimension of many different types of Lie algebras.

More specifically, for semisimple Lie algebras $\mathfrak{s}$ the invariant $\alpha(\mathfrak{s})$ was completely determined by Malcev [13]. Since there are no abelian ideals in $\mathfrak{s}$, we have $\beta(\mathfrak{s})=0$. The value of $\alpha$ for simple Lie algebras is reproduced in table 1. More recently much interest has focused on the study of abelian

Table 1: The invariant $\alpha$ for simple Lie algebras

\begin{tabular}{|c|c|c|}
\hline $\mathfrak{s}$ & $\operatorname{dim}(\mathfrak{s})$ & $\alpha(\mathfrak{s})$ \\
\hline$A_{n}, n \geq 1$ & $n(n+2)$ & $\left\lfloor\left(\frac{n+1}{2}\right)^{2}\right\rfloor$ \\
\hline$B_{3}$ & 21 & 5 \\
\hline$B_{n}, n \geq 4$ & $n(2 n+1)$ & $\frac{n(n-1)}{2}+1$ \\
\hline$C_{n}, n \geq 2$ & $n(2 n+1)$ & $\frac{n(n+1)}{2}$ \\
\hline$D_{n}, n \geq 4$ & $n(2 n-1)$ & $\frac{n(n-1)}{2}$ \\
\hline$G_{2}$ & 14 & 3 \\
\hline$F_{4}$ & 52 & 9 \\
\hline$E_{6}$ & 78 & 16 \\
\hline$E_{7}$ & 133 & 27 \\
\hline$E_{8}$ & 248 & 36 \\
\hline
\end{tabular}

ideals in a Borel subalgebra $\mathfrak{b}$ of a simple complex Lie algebra. It has been shown that $\alpha(\mathfrak{s})=\beta(\mathfrak{b})$, and this number can be computed purely in terms of certain root system invariants $([18])$. Furthermore, Kostant found a relationship between these invariants and discrete series representations of the corresponding Lie group, and to powers of the Euler product $([10,11]$. 
In fact,there are many more results concerning the invariants $\alpha$ and $\beta$ for simple Lie algebras and their Borel subalgebras.

We shall call $L$ supersolvable if there is a chain $0=L_{0} \subset L_{1} \subset \ldots \subset$ $L_{n-1} \subset L_{n}=L$, where $L_{i}$ is an $i$-dimensional ideal of $L$. The ideals $L^{(k)}$ of the derived series are defined by $L^{(0)}=L, L^{(k+1)}=\left[L^{(k)}, L^{(k)}\right]$ for $k \geq 0$; we also write $L^{2}$ for $L^{(1)}$ and $L^{3}$ for $\left[L^{2}, L\right]$. It is well known that every supersolvable Lie algebra is also solvable. Moreover, these classes coincide over an algebraically closed field of characteristic zero (Lie's theorem). There are, however, examples of solvable Lie algebras over algebraically closed field of non-zero characteristic which are not supersovable (see for instance $[9$, page 53] or [1]).

A number of authors have studied these invariants for solvable Lie algebras (see $[4,5,3,15]$ and the references contained therein). In particular, Burde and Ceballos showed that, for a solvable Lie algebra $L$ over an algebraically closed field of characteristic zero, $\alpha(L)=\beta(L)$ ([3]). They also gave an example to show that this was not true if the field was not algebraically closed. Nevertheless, it is worthwhile to explore the extent to which the algebraic closure and characteristic of the field are necessary for these two invariants to be equal. It would seem most likely that algebraic closure would be unnecessary for supersolvable Lie algebras. This was investigated by the author and Ceballos in [5] where, in particular it was shown that every supersolvable Lie algebra, $L$, of dimension $n$ with $\alpha(L)=n-2$ also satisfies $\beta(L)=n-2$ and that the $\alpha$ and $\beta$ invariants also coincide for nilpotent Lie algebras $L$ with $\alpha(L)=n-3$, provided that $F$ has characteristic different from two. An example is also given to show that this last result does not hold even over algebraically closed fields in characteristic two.

The current paper seeks to extend these results further; in particular answering question 1 in [5], and has the following structure. In section 2 we give some general results concerning maximal abelian subalgebras. In section 3 , we show that every supersolvable Lie algebra, $L$, of dimension $n$ with $\alpha(L)=n-3$ also satisfies $\beta(L)=n-3$ provided that $F$ has characteristic different from 2.. In the final section we show the $\alpha$ and $\beta$ invariants also coincide for nilpotent Lie algebras $L$ with $\alpha(L)=n-4$, provided that $F$ has characteristic greater than 5 .

\section{Some general results}

If $S$ is a subalgebra of $L$ we call the biggest ideal of $L$ contained in $S$ the core of $L$, and denote it by $S_{L}$; the smallest ideal of $L$ containing $S$ is called 
the ideal closure of $S$, and is denoted by $S^{L}$. Clearly $S^{L}=\sum_{i=0}^{\infty} S(\operatorname{ad} L)^{i}=$ $\sum_{i=0}^{r} S(\operatorname{ad} L)^{i}$ for some $r \in \mathbb{N}$. We will denote the centre of $L$ by $Z(L)=$ $\{x \in L:[x, y]=0, \forall y \in L\}$ and the centralizer of a subalgebra $A$ of $L$ by $C_{L}(A)=\{x \in L:[x, A]=0\}$. First we give some relationships between $A^{L}$ and $A_{L}$ when $A$ is an abelian subalgebra of $L$.

Proposition 2.1 Let $L$ be a Lie algebra over any field $F$ and let $A$ be an abelian subalgebra of $L$. Then $A_{L} \subseteq Z\left(A^{L}\right)$.

Proof. Suppose that $\left[A_{L}, A(\operatorname{ad} L)^{k}\right]=0$ for some $k \geq 0$. Then

$$
\begin{aligned}
{\left[A_{L}, A(\operatorname{ad} L)^{k+1}\right] } & =\left[A_{L},\left[A(\operatorname{ad} L)^{k}, L\right]\right] \\
& \subseteq\left[A(\operatorname{ad} L)^{k},\left[L, A_{L}\right]\right]+\left[L,\left[A_{L}, A(\operatorname{ad} L)^{k}\right]\right] \\
& =0 .
\end{aligned}
$$

Hence $\left[A_{L}, A^{L}\right]=0$ and $A_{L} \subseteq Z\left(A^{L}\right)$.

Corollary 2.2 Let $L$ be a Lie algebra over any field $F$ and let $A$ be a maximal abelian subalgebra of $L$. Then $A_{L}=Z\left(A^{L}\right)$.

Proof. This follows immediately, since $A+Z\left(A^{L}\right)$ is abelian and $Z\left(A^{L}\right)$ is an ideal of $L$.

Next we show that if $A$ is an abelian subalgebra which is an ideal of a maximal subalgebra of $L$ then $A^{L}$ is nilpotent. First we need a lemma.

Lemma 2.3 Let $S$ be a subalgebra of $L$ with $S^{L}=\sum_{i=0}^{r} S(a d L)^{i}$, and let $N$ be any ideal of $L$ in which $S$ is an ideal. Then $\sum_{i=0}^{k} S(a d L)^{i}$ is an ideal of $N$ for all $k=0, \ldots, r$.

Proof. Suppose that $\sum_{i=0}^{k} S(\operatorname{ad} L)^{i}$ is an ideal of $N$. Then

$$
\begin{aligned}
{\left[\sum_{i=0}^{k+1} S(\operatorname{ad} L)^{i}, N\right] } & =\left[\left[\sum_{i=0}^{k} S(\operatorname{ad} L)^{i}, L\right], N\right] \\
& \subseteq\left[[L, N], \sum_{i=0}^{k} S(\operatorname{ad} L)^{i}\right]+\left[\left[N, \sum_{i=0}^{k} S(\operatorname{ad} L)^{i}\right], L\right] \\
& \subseteq \sum_{i=0}^{k+1} S(\operatorname{ad} L)^{i} .
\end{aligned}
$$

Since $S$ is an ideal of $N$ the result follows from a simple induction. 
Theorem 2.4 Let $L$ be a Lie algebra over a field $F$, let $A$ be an abelian subalgebra of $L$ and let $N$ be an ideal of codimension one in $L$ in which $A$ is an ideal. Then

(i) $A^{L}=\sum_{i=0}^{r} A(a d x)^{i}$ for some $r \in \mathbb{N}$ and any $x \in L \backslash N$;

(ii) $\sum_{i=0}^{k} A(a d x)^{i}$ is a nilpotent ideal of $N$ of class $f(k)$ for $k=0, \ldots, r$, provided that, for $k \geq 1, F$ has characteristic zero or $p>f(k-1)+1$ where $f(k) \leq k(k+1)(2 k+1) / 6+2 k$; and

(iii) $A^{L}$ is a nilpotent ideal of $L$ of class $f(r)$ provided that $F$ has characteristic zero or $p>f(r-1)+1$.

\section{Proof.}

(i) It is clear that $L=N+F x$ and $\sum_{i=0}^{r} A(\operatorname{ad} x)^{i} \subseteq A^{L}$. But $\sum_{i=0}^{r} A(\operatorname{ad} x)^{i}$ is an ideal of $N$, by Lemma 3.1, and so of $L$. The reverse inclusion follows.

(ii) Clearly this is true for $k=0$. Suppose it holds for $k=m$ and put $I=\sum_{i=0}^{m} A(\operatorname{ad} x)^{i}$. Then $\sum_{i=0}^{m+1} A(\operatorname{ad} x)^{i}$ is an ideal of $N$, by Lemma 3.1. Moreover, $D=\left.\operatorname{ad} x\right|_{N}$ is a derivation of $N$, and so

$$
\left(\sum_{i=0}^{m+1} A(\operatorname{ad} x)^{i}\right)^{f(m+1)+1}=(I+D(I))^{f(m+1)+1}=0,
$$

provided that $F$ has characteristic zero or $p>f(m)+1$, by [12, Theorem 1].The result follows.

(iii) This is immediate from (ii).

As Maksimenko points out, the bound on $f(k)$ is rough and increases rapidly: $f(1)=3, f(2)=20$ and $f(3)=2910$. So this bound is unlikely to be particularly helpful in proving results.

Lemma 2.5 Let $L$ be a Lie algebra over a field $F$, let $A$ be a maximal abelian subalgebra of $L$ and suppose that $A^{L}$ is nilpotent and $\left(A^{L}\right)^{(2)}=0$. Then either

(i) $\left(A^{L}\right)^{3}=0$; or 
(ii) $\operatorname{dim}\left(\left(A^{L}\right)^{2}+Z\left(A^{L}\right)\right) / Z\left(A^{L}\right) \geq 2$.

Proof. First suppose that $\left(\left(A^{L}\right)^{2}+Z\left(A^{L}\right)\right) \cap A=Z\left(A^{L}\right)$. Then we have that $\left(A^{L}\right)^{2} \cap A+Z\left(A^{L}\right)=Z\left(A^{L}\right)$ and $\left(A^{L}\right)^{2} \cap A \subseteq Z\left(A^{L}\right)$. It follows that $\left[A^{L}, A\right] \subseteq Z\left(A^{L}\right)$, whence $\left[\left(A^{L}\right)^{2}, A\right] \subseteq\left[A^{L},\left[A^{L}, A\right]\right]=0$ and $\left(A^{L}\right)^{2}+A$ is an abelian subalgebra of $L$. The maximality of $A$ then yields that $\left(A^{L}\right)^{2} \subseteq A$, from which $\left(A^{L}\right)^{2} \subseteq A_{L}=Z\left(A^{L}\right)$ and $\left(A^{L}\right)^{3}=0$.

It follows from the above that if $(i)$ does not hold, then $\left(A^{L}\right)^{2} \nsubseteq A$ and $\left(\left(A^{L}\right)^{2}+Z\left(A^{L}\right)\right) \cap A \neq Z\left(A^{L}\right)$, which gives case $(i i)$.

Lemma 2.6 Let $L$ be a supersolvable Lie algebra and let $A$ be a maximal abelian subalgebra of $L$ for which $A^{L}=L$. Suppose that $A$ is an ideal of a maximal subalgebra $M$ of $L$. Then $L=L^{2} \dot{+} A$ where $\operatorname{dim} L^{2}=1$, and $\alpha(L)=\operatorname{dim} L-1=\beta(L)$.

Proof. We have immediately that $L=A^{L} \subseteq L^{2}+A$, so $L=L^{2}+A$. Let $K$ be a subspace of $A$ which is complementary to $L^{2}$ in $L$. Then $K$ is a subalgebra of $L=L^{2} \dot{+} K$ and $L^{2}$ is complemented in $L$. It follows from [20, Theorem 3.4] that

$$
\frac{L}{L^{(2)}}=\frac{L^{2}}{L^{(2)}} \dot{+} \frac{K+L^{(2)}}{L^{(2)}} \text { and } \frac{K+L^{(2)}}{L^{(2)}} \text { is a Cartan subalgebra of } \frac{L}{L^{(2)}} .
$$

As Cartan subalgebras are self-normalising we must have that $K+L^{(2)}=$ $A+L^{(2)}$ and $M+A+L^{(2)}=A+L^{(2)}$, whence $M \subseteq A+L^{(2)}$. But now $L^{2}$ is nilpotent, so $\phi\left(L^{2}\right)=L^{(2)} \subseteq \phi(L) \subseteq M$, by [19, Lemma 4.1], so $M=A+L^{(2)}$. Since $L$ is supersolvable, $M$ has codimension one in $L$ and so $L^{(2)}$ has codimension one in $L^{2}$. Hence $L^{2}=L^{(2)}+F x=\phi\left(L^{2}\right)+F x=F x$ for some $x \in L$. Hence $\operatorname{dim} L^{2}=1$ and $C_{L}\left(L^{2}\right)$ is an abelian ideal of codimension one in $L$. The result follows.

We will also need the following result, which is proved in [5, Lemma 2.5].

Lemma 2.7 Let $L$ be a supersolvable Lie algebra and let $A$ be a maximal abelian ideal of $L$. Then $C_{L}(A)=A$.

\section{Supersolvable Lie algebras of dimension $n$ with $\alpha(L)=n-3$}

. In this section we extend [5, Theorem 4.1] to supersolvable Lie algebras, thereby answering Question 1 in that paper. First we need the following Lemma which will be used several times. 
Lemma 3.1 Let $L$ be a supersolvable algebra over a field $F$ with an abelian subalgebra $A$ of maximal dimension. Let $A^{L}=F e_{2}+\ldots+F e_{m}+Z\left(A^{L}\right)$ $(m \geq 2)$. Suppose that $\operatorname{dim}\left(A^{L}\right)^{2}=r \leq m-2$. Then there exists an abelian ideal $B$ of $L$ of dimension $\operatorname{dim} Z\left(A^{L}\right)+1$ with $B \subseteq A^{L}$. Moreover, if $B$ is a maximal abelian ideal of $L$, then, for each set $\left\{e_{i_{1}}, \ldots, e_{i_{r+1}}\right\}$ of $r+1$ distinct elements of $\left\{e_{2}, \ldots, e_{m}\right\}$ there exist $\lambda_{i_{1}}, \ldots, \lambda_{i_{r+1}} \in F$, not all zero, such that

$$
B=Z\left(A^{L}\right)+\sum_{j=1}^{r+1} \lambda_{i_{j}} e_{i_{j}} .
$$

Proof. The ideal $B$ exists because $L$ is supersolvable and so there is a onedimensional ideal $B / Z\left(A^{L}\right)$ of $L / Z\left(A^{L}\right)$ with $B \subseteq A^{L}$. Put $B=F b+Z\left(A^{L}\right)$. Then $\left[e_{i_{1}}, b\right], \ldots,\left[e_{i_{r+1}}, b\right]$ are linearly dependent, since $\operatorname{dim}\left(A^{L}\right)^{2}=r$. Hence there there exist $\lambda_{i_{1}}, \ldots, \lambda_{i_{r+1}} \in F$, not all zero, such that

$$
\sum_{j=2}^{r+1} \lambda_{i_{j}}\left[e_{i_{j}}, b\right]=0 .
$$

If $B$ is a maximal abelian ideal of $L$ then $\sum_{j=2}^{r+1} \lambda_{i_{j}} e_{i_{j}} \in C_{L}(B)=B$, by Lemma 2.7.

Theorem 3.2 Let $L$ be a supersolvable Lie algebra over a field of characteristic different from 2 with an abelian subalgebra $A$ of maximal dimension. Suppose that $\alpha(L)=\operatorname{dim} A^{L}-2$, and that $A$ is an ideal of a maximal ideal $N$ of $L$. Then $\beta(L)=\operatorname{dim} A^{L}-2$.

Proof. Case A: Assume first that $A^{L}=F e_{2}+F e_{3}+A$ where $e_{3}=\left[e_{1}, e_{4}\right]$, $e_{2}=\left[e_{1}, e_{3}\right]$. By replacing $e_{j}$ by $e_{j}-\alpha_{j 2} e_{3}-\alpha_{j 3} e_{4}$ we can assume that $\left[e_{1}, e_{j}\right]=\sum_{i=4}^{n} \alpha_{j i} e_{i}$ for $5 \leq j \leq n$. In particular, this holds if $A+\left[A, e_{1}\right]$ is a subalgebra of codimension one in $A^{L}$, and $A^{L}=A+\left[A, e_{1}\right]+\left[\left[A, e_{1}\right], e_{1}\right]$. Then

$$
\left[e_{3}, e_{j}\right]=\left[\left[e_{1}, e_{4}\right], e_{j}\right]=-\left[\left[e_{4}, e_{j}\right], e_{1}\right]-\left[\left[e_{j}, e_{1}\right], e_{4}\right]=0 \text { for } j \geq 5
$$

Also

$$
\begin{aligned}
{\left[e_{2}, e_{j}\right]=\left[\left[e_{1}, e_{3}\right], e_{j}\right] } & =-\left[\left[e_{3}, e_{j}\right], e_{1}\right]-\left[\left[e_{j}, e_{1}\right], e_{3}\right] \\
& =\alpha_{j 4}\left[e_{4}, e_{3}\right] \text { for } j \geq 5
\end{aligned}
$$


If $\alpha_{k 4} \neq 0$ for some $k \geq 5$, interchange $e_{5}$ and $e_{k}$ and then replace $e_{j}$ by $\alpha_{54} e_{j}-\alpha_{j 4} e_{5}$ for $j \geq 6$. Then

$$
\begin{aligned}
& {\left[e_{2}, e_{j}\right]=0 \text { for } j \geq 6} \\
& {\left[e_{3}, e_{j}\right]=0 \text { for } j \geq 5 .}
\end{aligned}
$$

Hence $\operatorname{dim} Z\left(A^{L}\right) \geq \operatorname{dim} A^{L}-4$. Now

$$
\begin{aligned}
{\left[e_{1},\left[e_{3}, e_{4}\right]\right] } & =-\left[e_{3},\left[e_{4}, e_{1}\right]\right]-\left[e_{4},\left[e_{1}, e_{3}\right]\right]=\left[e_{2}, e_{4}\right], \text { and } \\
{\left[e_{1},\left[e_{2}, e_{5}\right]\right] } & =-\left[e_{2},\left[e_{5}, e_{1}\right]\right]-\left[e_{5},\left[e_{1}, e_{2}\right]\right] \\
& =\alpha_{54}\left[e_{2}, e_{4}\right]+\alpha_{55}\left[e_{2}, e_{5}\right]+\alpha_{22}\left[e_{2}, e_{5}\right] .
\end{aligned}
$$

But $\left[e_{2}, e_{5}\right]=\alpha_{54}\left[e_{4}, e_{3}\right]$, so (2), (5) and (6) yield

$$
2 \alpha_{54}\left[e_{2}, e_{4}\right]=\left(\alpha_{55}+\alpha_{22}\right)\left[e_{5}, e_{2}\right]=\alpha_{54}\left(\alpha_{55}+\alpha_{22}\right)\left[e_{3}, e_{4}\right] .
$$

If $\alpha_{54}=0$ we have that $\left[e_{2}, e_{5}\right]=0, \operatorname{so} \operatorname{dim} Z\left(A^{L}\right) \geq \operatorname{dim} A^{L}-3$ and Lemma 3.1 implies that $\beta(L)=\operatorname{dim} A^{L}-2$.

So suppose that $\alpha_{54} \neq 0$. Then $\left(A^{L}\right)^{2}$ is spanned by $\left[e_{2}, e_{3}\right]$ and $\left[e_{3}, e_{4}\right]$, whence $\operatorname{dim}\left(A^{L}\right)^{2} \leq 2$. Lemma 3.1 implies that there is an abelian ideal $B$ of $L$ of dimension $\operatorname{dim} A^{L}-3$ inside $A^{L}$ and $B=\lambda_{3} e_{3}+\lambda_{4} e_{4}+\lambda_{5} e_{5}+Z\left(A^{L}\right)=$ $\mu_{2} e_{2}+\mu_{4} e_{4}+\mu_{5} e_{5}+Z\left(A^{L}\right)$ for some $\lambda_{3}, \lambda, \lambda_{5}, \mu_{2}, \mu_{4}, \mu_{5} \in F$ and where not all of the $\lambda^{\prime} s$ or $\mu^{\prime} s$ are zero. Thus $\lambda_{3}=\mu_{2}=0$ and $B \subseteq A$. If $B$ is a maximal abelian ideal of $L$ we have $e_{4}, e_{5} \in C_{L}(B)=B$, a contradiction. Hence $\beta(L)=\operatorname{dim} A^{L}-2$.

Case B Suppose now that $A^{L}=A+\left[A, e_{1}\right]$; we can assume that $A^{L}=$ $F e_{2}+F e_{3}+A$ where $e_{2}=\left[e_{1}, e_{4}\right], e_{3}=\left[e_{1}, e_{5}\right]$. Again, by replacing $e_{j}$ by $e_{j}-\alpha_{j 2} e_{4}-\alpha_{j 3} e_{5}$, we can assume that $\left[e_{1}, e_{j}\right]=\sum_{i=4}^{n} \alpha_{j i} e_{i}$ for $6 \leq j \leq n$. Then,

$$
\begin{aligned}
& {\left[e_{2}, e_{j}\right]=\left[\left[e_{1}, e_{4}\right], e_{j}\right]=-\left[\left[e_{4}, e_{j}\right], e_{1}\right]-\left[\left[e_{j}, e_{1}\right], e_{4}\right]=0 \text { for } j \geq 6,} \\
& {\left[e_{2}, e_{5}\right]=\left[e_{3}, e_{4}\right] \text { and }} \\
& {\left[e_{3}, e_{j}\right]=\left[\left[e_{1}, e_{5}\right], e_{j}\right]=-\left[\left[e_{5}, e_{j}\right], e_{1}\right]-\left[\left[e_{j}, e_{1}\right], e_{5}\right]=0 \text { for } j \geq 6 .}
\end{aligned}
$$

Hence $\operatorname{dim} Z\left(A^{L}\right) \geq 4$.

If $\alpha_{32} \neq 0$ then replace $e_{2}$ by $\left[e_{1}, e_{3}\right]$ and we are in Case A. Similarly, if $\alpha_{23} \neq 0$ then replace $e_{3}$ by $\left[e_{1}, e_{2}\right]$ and we are in Case $\mathrm{A}$ again. So we can assume that $\alpha_{23}=\alpha_{32}=0$. Now

$$
\begin{aligned}
{\left[e_{1},\left[e_{2}, e_{5}\right]\right] } & =-\left[e_{2},\left[e_{5}, e_{1}\right]\right]-\left[e_{5},\left[e_{1}, e_{2}\right]\right] \\
& =\left[e_{2}, e_{3}\right]+\alpha_{22}\left[e_{2}, e_{5}\right] \\
{\left[e_{1},\left[e_{3}, e_{4}\right]\right] } & =-\left[e_{3},\left[e_{4}, e_{1}\right]\right]-\left[e_{4},\left[e_{1}, e_{3}\right]\right] \\
& =\left[e_{3}, e_{2}\right]+\alpha_{33}\left[e_{3}, e_{4}\right]
\end{aligned}
$$


Then (9),(11) and (12) imply that

$$
2\left[e_{2}, e_{3}\right]=\alpha_{33}\left[e_{3}, e_{4}\right]-\alpha_{22}\left[e_{2}, e_{5}\right]=\left(\alpha_{33}-\alpha_{22}\right)\left[e_{3}, e_{4}\right]
$$

Now,

$$
\begin{aligned}
{\left[e_{1},\left[e_{2}, e_{3}\right]\right]=} & -\left[e_{2},\left[e_{3}, e_{1}\right]\right]-\left[e_{3},\left[e_{1}, e_{2}\right]\right] \\
= & \left(\alpha_{22}+\alpha_{33}\right)\left[e_{2}, e_{3}\right]+\left(\alpha_{35}-\alpha_{24}\right)\left[e_{3}, e_{4}\right]+\alpha_{34}\left[e_{2}, e_{4}\right] \\
& +\alpha_{25}\left[e_{5}, e_{3}\right]
\end{aligned}
$$

Then (13),(14) imply that

$$
\begin{array}{r}
2\left(\alpha_{22}+\alpha_{33}\right)\left[e_{2}, e_{3}\right]+2\left(\alpha_{35}-\alpha_{24}\right)\left[e_{3}, e_{4}\right]+2 \alpha_{34}\left[e_{2}, e_{4}\right]+2 \alpha_{25}\left[e_{5}, e_{3}\right] \\
=\left(\alpha_{33}-\alpha_{22}\right)\left[e_{3}, e_{2}\right]+\alpha_{33}\left(\alpha_{33}-\alpha_{22}\right)\left[e_{3}, e_{4}\right]
\end{array}
$$

which yields

$$
\left(\left(\alpha_{33}^{2}-\alpha_{22}^{2}\right)+4\left(\alpha_{35}-\alpha_{24}\right)\right)\left[e_{3}, e_{4}\right]+4 \alpha_{34}\left[e_{2}, e_{4}\right]+4 \alpha_{25}\left[e_{5}, e_{3}\right]=0 .
$$

If at least one of the coefficients in (16) is non-zero, then $\operatorname{dim}\left(A^{L}\right)^{2} \leq 2$ and we can argue as in the last paragraph before Case B.

So suppose that all of the coefficients in (16) are all zero; that is,

$$
\alpha_{34}=0, \quad \alpha_{25}=0, \quad \alpha_{33}^{2}+4 \alpha_{35}=\alpha_{22}^{2}+4 \alpha_{24} .
$$

Using Lemma 3.1 there is an abelian ideal $B=\sum_{i=2}^{5} \lambda_{i} e_{i}+Z\left(A^{L}\right)$ where not all of the $\lambda^{\prime} s$ are zero. Now

$$
\begin{aligned}
& {\left[e_{1}, \lambda_{2} e_{2}+\lambda_{3} e_{3}+\lambda_{4} e_{4}+\lambda_{5} e_{5}\right]} \\
& =\lambda_{2}\left(\alpha_{22} e_{2}+\alpha_{24} e_{4}\right)+\lambda_{3}\left(\alpha_{33} e_{3}+\alpha_{35} e_{5}\right)+\lambda_{4} e_{2}+\lambda_{5} e_{3} \\
& =k\left(\lambda_{2} e_{2}+\lambda_{3} e_{3}+\lambda_{4} e_{4}+\lambda_{5} e_{5}+z\right)
\end{aligned}
$$

for some $k \in F, z \in Z\left(A^{L}\right)$. This yields

$$
\begin{aligned}
\lambda_{2} \alpha_{22}+\lambda_{4} & =k \lambda_{2} \\
\lambda_{3} \alpha_{33}+\lambda_{5} & =k \lambda_{3} \\
\lambda_{2} \alpha_{24} & =k \lambda_{4} \\
\lambda_{3} \alpha_{35} & =k \lambda_{5}
\end{aligned}
$$

Substituting (20) into $k \times(18)$ gives $\lambda_{2}\left(k^{2}-\alpha_{22} k-\alpha_{24}\right)=0$. Similarly, substituting (21) into $k \times(19)$ gives $\lambda_{3}\left(k^{2}-\alpha_{33} k-\alpha_{35}\right)=0$. If $\lambda_{2}=0$ 
then $\lambda_{4}=0$ and $B=\lambda_{3} e_{3}+\lambda_{5} e_{5}+Z\left(A^{L}\right)$. We must have $\lambda_{3} \neq 0$, since, otherwise, $\lambda_{5}=0$ also. If $\lambda_{5} \neq 0$ then $\lambda_{3} e_{3}-\lambda_{5} e_{5} \in C_{L}(B)$, so $B$ is not a maximal abelian ideal of $L$ and $\beta(L)=\operatorname{dim} A^{L}-2$. If $\lambda_{5}=0$, then $B=F e_{3}+Z\left(A^{L}\right)$ and (13) implies that $2 e_{2}+\left(\alpha_{33}-\alpha_{22}\right) e_{4} \in C_{L}(B)$ and so $B$ is not maximal again.

So assume that $\lambda_{2} \neq 0$. Similarly we can assume that $\lambda_{3} \neq 0$. Thus

$$
k=\frac{\alpha_{22} \pm \sqrt{\alpha_{22}^{2}+4 \alpha_{24}}}{2}=\frac{\alpha_{33} \pm \sqrt{\alpha_{33}^{2}+4 \alpha_{35}}}{2} .
$$

But now (17) implies that $\alpha_{22}=\alpha_{33}$ and $\alpha_{24}=\alpha_{35}$. Also, (18), (19) give $\lambda_{4}=\mu \lambda_{2}$ and $\lambda_{5}=\mu \lambda_{3}$, where $\mu=k-\alpha_{22}$, so

$$
B=\lambda_{2}\left(e_{2}+\mu e_{4}\right)+\lambda_{3}\left(e_{3}+\mu e_{5}\right) .
$$

But now $\left(e_{2}+\mu e_{4}\right),\left(e_{3}+\mu e_{5}\right) \in C_{L}(B)$ by (9) and (13). It follows that $B$ cannot be a maximal abelian ideal of $L$, completing the proof.

Corollary 3.3 Let $L$ be a supersolvable Lie algebra of dimension n, over a field $F$ of characteristic different from 2 , with $\alpha(L)=n-3$. Then $\beta(L)=$ $n-3$.

Proof. Let $A$ be an abelian subalgebra of $L$ with $\operatorname{dim} A=n-3$. Then $A^{L} \neq L$ by Lemma 2.6. Let $N$ be a maximal subalgebra of $L$ containing $A^{L}$. We can suppose that $A$ is an ideal of $N$, by [5, Corollary 3.6]. If $A^{L} \neq N$, then $A$ has codimension one in $A^{L}$ and the result is given by [5, Corollary 3.2]. So suppose now that $N=A^{L}$. Then $\alpha(L)=\operatorname{dim} A^{L}-2$ and the result is given by Theorem 3.2 .

\section{Nilpotent Lie algebras of dimension $n$ with $\alpha(L)=$ $n-4$}

Here we show that when $L$ is nilpotent and $\alpha(L)=n-4$ then $\beta(L)=n-4$ provided that $F$ has characteristic different from 2,3 and 5 .

Theorem 4.1 Let $L$ be a nilpotent Lie algebra of dimension $n$ over a field $F$ of characteristic different from $2,3,5$ with $\alpha(L)=n-4$. Then $\beta(L)=n-4$ also. 
Proof. Let $A$ be an abelian subalgebra of $L$ with $\operatorname{dim} A=n-4$, let $N$ be a maximal subalgebra of $L$ containing $A$ and suppose that $A$ is not an ideal of $L$. Then $N$ is an ideal of $L$ and $A$ is an abelian subalgebra of $N$ of maximal dimension and codimension 3 in $N$. By Theorem 3.2 we can assume $A$ is an ideal of $N$. Furthermore, we can assume that $N=A^{L}$, so that $Z(N)=A_{L}$. Let $e_{5}, \ldots, e_{n}$ be a basis for $A$, and let $L=F e_{1}+N$. We consider three cases.

Case A: Suppose first that $N=F e_{2}+F e_{3}+F e_{4}+A$ where $e_{4}=\left[e_{1}, e_{5}\right]$, $e_{3}=\left[e_{1}, e_{4}\right], e_{2}=\left[e_{1}, e_{3}\right]$. In particular, this is the case if $A+\left[A, e_{1}\right]$ is a subalgebra of codimension two in $N$, so that $N=A+\left[A, e_{1}\right]+\left[\left[A, e_{1}\right], e_{1}\right]+$ $\left[\left[\left[A, e_{1}\right], e_{1}\right], e_{1}\right]$. Relpacing $e_{j}$ by $e_{j}-\alpha_{j 2} e_{3}-\alpha_{j 3} e_{4}-\alpha_{j 4} e_{5}$ we can assume that $\left[e_{1}, e_{j}\right]=\sum_{i=5}^{n} \alpha_{j i} e_{i}$ for $j \geq 6$. Then

$$
\begin{aligned}
{\left[e_{4}, e_{j}\right] } & =\left[\left[e_{1}, e_{5}\right], e_{j}\right]=-\left[\left[e_{5}, e_{j}\right] e_{1}\right]-\left[\left[e_{j}, e_{1}\right], e_{5}\right] \\
& =0 \text { for } j \geq 6 .
\end{aligned}
$$

Also

$$
\begin{aligned}
{\left[e_{3}, e_{j}\right] } & =\left[\left[e_{1}, e_{4}\right], e_{j}\right]=-\left[\left[e_{4}, e_{j}\right], e_{1}\right]-\left[\left[e_{j}, e_{1}\right], e_{4}\right] \\
& =\alpha_{j 5}\left[e_{5}, e_{4}\right] \text { for } j \geq 6
\end{aligned}
$$

If $\alpha_{k 5} \neq 0$ for some $k \geq 6$, interchange $e_{6}$ and $e_{k}$ and then replace $e_{j}$ by $\alpha_{65} e_{j}-\alpha_{j 5} e_{6}$ for $j \geq 7$, so we can assume that

$$
\left[e_{3}, e_{j}\right]=\left[e_{4}, e_{j}\right]=0 \text { and } \alpha_{j 5}=0 \text { for } j \geq 7
$$

Moreover,

$$
\begin{aligned}
{\left[e_{2}, e_{j}\right] } & =\left[\left[e_{1}, e_{3}\right], e_{j}\right]=-\left[\left[e_{3}, e_{j}\right], e_{1}\right]-\left[\left[e_{j}, e_{1}\right], e_{3}\right] \\
& =\alpha_{j 6}\left[e_{6}, e_{3}\right] \text { for } j \geq 7
\end{aligned}
$$

If $\alpha_{k 6} \neq 0$ for some $k \geq 7$, interchange $e_{7}$ and $e_{k}$ and then replace $e_{j}$ by $\alpha_{76} e_{j}-\alpha_{j 6} e_{7}$ for $j \geq 7$, so we can assume that

$$
\left[e_{2}, e_{j}\right]=0 \text { and } \alpha_{j 6}=0 \text { for } j \geq 8,
$$

as before.

Now

$$
\begin{aligned}
{\left[e_{1},\left[e_{3}, e_{6}\right]\right] } & =-\left[e_{3},\left[e_{6}, e_{1}\right]\right]-\left[e_{6},\left[e_{1}, e_{3}\right]\right] \\
& =\alpha_{65}\left[e_{3}, e_{5}\right]+\alpha_{66}\left[e_{3}, e_{6}\right]+\left[e_{2}, e_{6}\right] \\
{\left[e_{1},\left[e_{5}, e_{4}\right]\right] } & =-\left[e_{5},\left[e_{4}, e_{1}\right]\right]-\left[e_{4},\left[e_{1}, e_{5}\right]\right]=\left[e_{5}, e_{3}\right], \text { so } \\
{\left[e_{2}, e_{6}\right] } & =2 \alpha_{65}\left[e_{5}, e_{3}\right]+\alpha_{66}\left[e_{6}, e_{3}\right] \text { from }(24)
\end{aligned}
$$


Moreover

$$
\begin{aligned}
{\left[e_{1},\left[e_{2}, e_{6}\right]\right] } & =-\left[e_{2},\left[e_{6}, e_{1}\right]\right]-\left[e_{6},\left[e_{1}, e_{2}\right]\right] \\
& =\alpha_{65}\left[e_{2}, e_{5}\right]+\alpha_{66}\left[e_{2}, e_{6}\right]+\alpha_{67}\left[e_{2}, e_{7}\right]+\alpha_{22}\left[e_{2}, e_{6}\right] \\
& +\alpha_{23}\left[e_{3}, e_{6}\right] \\
{\left[e_{1},\left[e_{5}, e_{3}\right]\right] } & =-\left[e_{5},\left[e_{3}, e_{1}\right]\right]-\left[e_{3},\left[e_{1}, e_{5}\right]\right] \\
& =\left[e_{5}, e_{2}\right]+\left[e_{4}, e_{3}\right] \text { so } \\
2 \alpha_{65}\left[e_{3}, e_{4}\right] & =2 \alpha_{65}\left[e_{5}, e_{2}\right]-\alpha_{66}\left(\alpha_{65}\left[e_{3}, e_{5}\right]+\alpha_{66}\left[e_{3}, e_{6}\right]+\left[e_{2}, e_{6}\right]\right) \\
& +\alpha_{65}\left[e_{5}, e_{2}\right]+\alpha_{66}\left[e_{6}, e_{2}\right]+\alpha_{67}\left[e_{7}, e_{2}\right]+\alpha_{22}\left[e_{6}, e_{2}\right] \\
& +\alpha_{23}\left[e_{6}, e_{3}\right] \\
& =3 \alpha_{65}\left[e_{5}, e_{2}\right]+\alpha_{66} \alpha_{65}\left[e_{5}, e_{3}\right]+\left(\alpha_{66}^{2}+\alpha_{23}\right)\left[e_{6}, e_{3}\right] \\
& +\left(2 \alpha_{66}+\alpha_{22}\right)\left[e_{6}, e_{2}\right]+\alpha_{67}\left[e_{7}, e_{2}\right]
\end{aligned}
$$

If $\alpha_{65}=0$ we have that $\sum_{i=6}^{n} F e_{i}$ is an ideal of $L$ and so is contained in $Z(N)$. But now $L$ has an abelian ideal of dimension $\operatorname{dim} N-3$. So assume that $\alpha_{65} \neq 0$. Then equations (24), (25), (26), (27), (28) and (29) imply that $N^{2}$ is spanned by $\left[e_{2}, e_{3}\right],\left[e_{2}, e_{4}\right],\left[e_{2}, e_{5}\right],\left[e_{3}, e_{5}\right]$ and $\left[e_{4}, e_{5}\right]$, and so has dimension at most five.

Case A1 Suppose that $e_{7} \in Z(N)$, so that $\operatorname{dim}(N / Z(N) \leq 5$. Then $4 \geq$ $\operatorname{dim}([L, N]+Z(N)) \geq 3$, since $e_{2}, e_{3}, e_{4} \in[L, N]+Z(N)$.

(i) Suppose first that $\operatorname{dim}([L, N]+Z(N))=3$, so $\alpha_{25}=\alpha_{26}=\alpha_{45}=\alpha_{46}=0$. Then $\left[e_{1}, e_{2}\right] \in[L,[L,[L,[L, N]]]]+Z(N)=0$, so $\alpha_{22}=\alpha_{23}=\alpha_{24}=0$ and $\mathrm{Fe}_{2}+Z(N)$ is an abelian ideal of dimension $\operatorname{dim} N-4$. Then

$$
\begin{aligned}
{\left[e_{1},\left[e_{2}, e_{3}\right]\right] } & =-\left[e_{2},\left[e_{3}, e_{1}\right]\right]-\left[e_{3},\left[e_{1}, e_{2}\right]\right]=0 \\
{\left[e_{1},\left[e_{2}, e_{4}\right]\right] } & =-\left[e_{2},\left[e_{4}, e_{1}\right]\right]-\left[e_{4},\left[e_{1}, e_{2}\right]\right] \\
& =\left[e_{2}, e_{3}\right] \\
{\left[e_{1},\left[e_{2}, e_{5}\right]\right] } & =-\left[e_{2},\left[e_{5}, e_{1}\right]\right]-\left[e_{5},\left[e_{1}, e_{2}\right]\right] \\
& =\left[e_{2}, e_{4}\right] \\
{\left[e_{1},\left[e_{2}, e_{6}\right]\right] } & =-\left[e_{2},\left[e_{6}, e_{1}\right]\right]-\left[e_{6},\left[e_{1}, e_{2}\right]\right] \\
& =\alpha_{65}\left[e_{2}, e_{5}\right]+\alpha_{66}\left[e_{2}, e_{6}\right]
\end{aligned}
$$

Hence, $F\left[e_{2}, e_{3}\right]+F\left[e_{2}, e_{4}\right]+F\left[e_{2}, e_{5}\right]+F\left[e_{2}, e_{6}\right]$ is an ideal of $L$ of dimension at most 4 . It follows that

$$
\begin{aligned}
0 & =\left[e_{1},\left[e_{1},\left[e_{1},\left[e_{1},\left[e_{2}, e_{6}\right]\right]\right]\right]\right] \\
& =\alpha_{66} \alpha_{65}\left[e_{2}, e_{3}\right]+\alpha_{66}^{2} \alpha_{65}\left[e_{2}, e_{4}\right]+\alpha_{66}^{3} \alpha_{65}\left[e_{2}, e_{5}\right]+\alpha_{66}^{4}\left[e_{2}, e_{6}\right]
\end{aligned}
$$


If $\alpha_{66} \neq 0$ we have that $\alpha_{65} e_{3}+\alpha_{66} \alpha_{65} e_{4}+\alpha_{66}^{2} \alpha_{65} e_{5}+\alpha_{66}^{3} e_{6} \in C_{L}\left(F e_{2}+\right.$ $Z(N))$ and $L$ has an abelian ideal of dimension $\operatorname{dim} N-3$.

So suppose that $\alpha_{66}=0$. Then (29) gives $2 \alpha_{65}\left[e_{3}, e_{4}\right]=3 \alpha_{65}\left[e_{5}, e_{2}\right]$. Multiplying both sides by $e_{1}$ then yields $2 \alpha_{65}\left[e_{2}, e_{4}\right]=3 \alpha_{65}\left[e_{4}, e_{2}\right]$, whence $5 \alpha_{65}\left[e_{2}, e_{4}\right]=0$. If $\alpha_{65}=0$ there is an ideal of dimension $\operatorname{dim} N-3$, as in the paragraph immediately preceeding Case A1. If $\alpha_{65} \neq 0$ then $e_{4} \in$ $C_{L}\left(F e_{2}+Z(N)\right)$ and again $L$ has an abelian ideal of dimension $\operatorname{dim} N-3$. (ii) So suppose that $\operatorname{dim}([L, N]+Z(N))=4$. Then there is an $n_{1} \in N$ such that $N=F n_{1}+F n_{2}+F n_{3}+F n_{4}+F n_{5}+Z(N)$ where $n_{2}=\left[e_{1}, n_{1}\right]$, $n_{3}=\left[e_{1}, n_{2}\right], n_{4}=\left[e_{1}, n_{3}\right], n_{5}=\left[e_{1}, n_{4}\right]$ and $\left[e_{1}, n_{5}\right] \in Z(N)$. Now

$$
\begin{aligned}
& {\left[e_{1},\left[n_{1}, n_{2}\right]\right]=-\left[n_{1},\left[n_{2}, e_{1}\right]\right]-\left[n_{2},\left[e_{1}, n_{1}\right]\right]=\left[n_{1}, n_{3}\right]} \\
& {\left[e_{1},\left[n_{1}, n_{3}\right]\right]=-\left[n_{1},\left[n_{3}, e_{1}\right]\right]-\left[n_{3},\left[e_{1}, n_{1}\right]\right]=\left[n_{1}, n_{4}\right]+\left[n_{2}, n_{3}\right]} \\
& {\left[e_{1},\left[n_{1}, n_{4}\right]\right]=-\left[n_{1},\left[n_{4}, e_{1}\right]\right]-\left[n_{4},\left[e_{1}, n_{1}\right]\right]=\left[n_{1}, n_{5}\right]+\left[n_{2}, n_{4}\right]} \\
& {\left[e_{1},\left[n_{2}, n_{3}\right]\right]=-\left[n_{2},\left[n_{3}, e_{1}\right]\right]-\left[n_{3},\left[e_{1}, n_{2}\right]\right]=\left[n_{2}, n_{4}\right]} \\
& {\left[e_{1},\left[n_{1}, n_{5}\right]\right]=-\left[n_{1},\left[n_{5}, e_{1}\right]\right]-\left[n_{5},\left[e_{1}, n_{1}\right]\right]=\left[n_{2}, n_{5}\right]} \\
& {\left[e_{1},\left[n_{2}, n_{4}\right]\right]=-\left[n_{2},\left[n_{4}, e_{1}\right]\right]-\left[n_{4},\left[e_{1}, n_{2}\right]\right]=\left[n_{2}, n_{5}\right]+\left[n_{3}, n_{4}\right]} \\
& {\left[e_{1},\left[n_{2}, n_{5}\right]\right]=-\left[n_{2},\left[n_{5}, e_{1}\right]\right]-\left[n_{5},\left[e_{1}, n_{2}\right]\right]=\left[n_{3}, n_{5}\right]} \\
& {\left[e_{1},\left[n_{3}, n_{4}\right]\right]=-\left[n_{3},\left[n_{4}, e_{1}\right]\right]-\left[n_{4},\left[e_{1}, n_{3}\right]\right]=\left[n_{3}, n_{5}\right]}
\end{aligned}
$$

Since $\operatorname{dim} N^{2} \leq 5$,

$$
0=\left[e_{1},\left[e_{1},\left[e_{1},\left[e_{1},\left[e_{1},\left[n_{1}, n_{2}\right]\right]\right]\right]\right]\right]=5\left[n_{3}, n_{5}\right]
$$

Clearly $F n_{5}+Z(N)$ is an abelian ideal of $L$ and $n_{3} \in C_{L}\left(F n_{5}+Z(N)\right.$, so $L$ has an abelian ideal of dimension $\operatorname{dim} N-3$.

Case A2 If $\alpha_{76}=0$ we are in Case A1. So suppose that $\alpha_{76} \neq 0$. Then $\left[e_{2}, e_{7}\right]=\alpha_{76}\left[e_{6}, e_{3}\right]$ Multiplying both sides of this by $e_{1}$ gives

$$
\begin{aligned}
{\left[e_{1},\left[e_{2}, e_{7}\right]\right] } & =-\left[e_{2},\left[e_{7}, e_{1}\right]\right]-\left[e_{7},\left[e_{1}, e_{2}\right]\right] \\
& =\alpha_{76}\left[e_{2}, e_{6}\right]+\alpha_{77}\left[e_{2}, e_{7}\right]+\alpha_{22}\left[e_{2}, e_{7}\right] \\
& =2 \alpha_{76} \alpha_{65}\left[e_{5}, e_{3}\right]+\left(\alpha_{66}+\alpha_{77}+\alpha_{22}\right) \alpha_{76}\left[e_{6}, e_{3}\right] \text { by }(28) \\
& =\alpha_{76}\left[e_{1},\left[e_{6}, e_{3}\right]\right] \\
& =\alpha_{76} \alpha_{65}\left[e_{5}, e_{3}\right]+\alpha_{76} \alpha_{66}\left[e_{6}, e_{3}\right]+\alpha_{76}\left[e_{6}, e_{2}\right] \\
& =\alpha_{76} \alpha_{65}\left[e_{5}, e_{3}\right]+\alpha_{76} \alpha_{66}\left[e_{6}, e_{3}\right]+2 \alpha_{76} \alpha_{65}\left[e_{3}, e_{5}\right]+\alpha_{76} \alpha_{66}\left[e_{3}, e_{6}\right] \\
& =\alpha_{76} \alpha_{65}\left[e_{3}, e_{5}\right]
\end{aligned}
$$

Hence

$$
3 \alpha_{65}\left[e_{5}, e_{3}\right]=\left(\alpha_{66}+\alpha_{77}+\alpha_{22}\right)\left[e_{3}, e_{6}\right] .
$$


If $\alpha_{65}=0$ we can argue as in the last paragraph before Case A1, so assume that $\alpha_{65} \neq 0$. Then we have that $\operatorname{dim} N^{2} \leq 4$. Suppose that $B=F b+Z(N)$ is a maximal abelian ideal of $L$. We have, by Lemma 3.1, that there exist $\lambda_{i} \in F$, not all zero, such that $\sum_{i=3}^{7} \lambda_{i} e_{i} \in C_{L}(B)=B$. But now $e_{7} \in Z(N)$ and we are in Case A1.

There are two further cases.

Case B: Suppose that $N=F e_{2}+F e_{3}+F e_{4}+A$ where $e_{4}=\left[e_{1}, e_{6}\right]$, $e_{3}=\left[e_{1}, e_{5}\right], e_{2}=\left[e_{1}, e_{3}\right]$. In particular, this is the case if $A+\left[A, e_{1}\right]$ is a subalgebra of codimension one in $N$, and $N=A+\left[A, e_{1}\right]+\left[\left[A, e_{1}\right], e_{1}\right]$ with $e_{3}, e_{4}, A$ spanning $\left[A, e_{1}\right]+A$ and $e_{2}, A$ spanning $\left[\left[A, e_{1}\right], e_{1}\right]+A$. Replacing $e_{j}$ by $e_{j}-\alpha_{j 2} e_{3}-\alpha_{j 3} e_{5}-\alpha_{j 4} e_{6}$ for $j \geq 7$, we can assume that $\left[e_{1}, e_{j}\right]=$ $\sum_{i=2}^{n} \alpha_{j i} e_{i}$ for $2 \leq j \leq 4$, and $\left[e_{1}, e_{j}\right]=\sum_{i=5}^{n} \alpha_{j i} e_{i}$ for $7 \leq j \leq n$. Then,

$$
\begin{aligned}
& \qquad \begin{aligned}
{\left[e_{3}, e_{j}\right] } & =\left[\left[e_{1}, e_{5}\right], e_{j}\right]=-\left[\left[e_{5}, e_{j}\right], e_{1}\right]-\left[\left[e_{j}, e_{1}\right], e_{5}\right]=0 \\
& \text { for } j \geq 7, \\
{\left[e_{4}, e_{j}\right] } & =\left[\left[e_{1}, e_{6}\right], e_{j}\right]=-\left[\left[e_{6}, e_{j}\right], e_{1}\right]-\left[\left[e_{j}, e_{1}\right], e_{6}\right]=0 \\
& \text { for } j \geq 7,
\end{aligned} \\
& \text { and } \quad\left[e_{3}, e_{6}\right]=\left[e_{4}, e_{5}\right] .
\end{aligned}
$$

Also

$$
\begin{aligned}
{\left[e_{2}, e_{j}\right]=\left[\left[e_{1}, e_{3}\right], e_{j}\right] } & =-\left[\left[e_{3}, e_{j}\right], e_{1}\right]-\left[\left[e_{j}, e_{1}\right], e_{3}\right] \\
& =\alpha_{j 5}\left[e_{5}, e_{3}\right]+\alpha_{j 6}\left[e_{6}, e_{3}\right] \text { for } j \geq 7
\end{aligned}
$$

If $\alpha_{k 5} \neq 0$ for some $k \geq 7$, interchange $e_{7}$ and $e_{k}$ and then replace $e_{j}$ by $\alpha_{75} e_{j}-\alpha_{j 5} e_{7}$ for $j \geq 8$, so we can assume that

$$
\left[e_{2}, e_{j}\right]=\alpha_{j 6}\left[e_{6}, e_{3}\right] \text { and } \alpha_{j 5}=0 \text { for } j \geq 8 \text {. }
$$

Similarly, if $\alpha_{k 6} \neq 0$ for some $k \geq 8$, interchange $e_{8}$ and $e_{k}$ and then replace $e_{j}$ by $\alpha_{86} e_{j}-\alpha_{j 6} e_{8}$ for $j \geq 9$, so we can assume that

$$
\left[e_{2}, e_{j}\right]=0 \text { and } \alpha_{j 6}=0 \text { for } j \geq 9 .
$$

Hence $\operatorname{dim} Z(N) \geq \operatorname{dim} N-7$.

Replacing $e_{4}$ by $e_{4}-\alpha_{42} e_{3}$ and $e_{6}$ by $e_{6}-\alpha_{42} e_{5}$ we can assume that $\alpha_{42}=0$.

Claim: $N^{2}$ is spanned by $z_{1}=\left[e_{2}, e_{5}\right], z_{2}=\left[e_{3}, e_{4}\right], z_{3}=\left[e_{3}, e_{5}\right], z_{4}=$ $\left[e_{3}, e_{6}\right]$ and $z_{5}=\left[e_{4}, e_{6}\right]$. In particular, $N^{3}=0$. 
Put $S=\sum_{i=1}^{5} F z_{i}$. It suffices to check $\left[e_{2}, e_{3}\right],\left[e_{2}, e_{4}\right]$ and $\left[e_{2}, e_{6}\right]$; the rest are covered by (30)-(35). Now,

$$
\begin{aligned}
{\left[e_{1},\left[e_{3}, e_{6}\right]\right] } & =-\left[e_{3},\left[e_{6}, e_{1}\right]\right]-\left[e_{6},\left[e_{1}, e_{3}\right]\right]=\left[e_{3}, e_{4}\right]+\left[e_{2} . e_{6}\right] \text { and } \\
{\left[e_{1},\left[e_{4}, e_{5}\right]\right] } & =-\left[e_{4},\left[e_{5}, e_{1}\right]\right]-\left[e_{5},\left[e_{1}, e_{4}\right]\right] \\
& =\left[e_{4}, e_{3}\right]+\alpha_{43}\left[e_{3}, e_{5}\right]+\alpha_{44}\left[e_{4}, e_{5}\right] \\
\text { so }\left[e_{2}, e_{6}\right] & =2\left[e_{4}, e_{3}\right]+\alpha_{43}\left[e_{3}, e_{5}\right]+\alpha_{44}\left[e_{3}, e_{6}\right] \text { by }(32)
\end{aligned}
$$

which gives that $\left[e_{2}, e_{6}\right] \in S$. Also

$$
\begin{aligned}
{\left[e_{1},\left[e_{2}, e_{6}\right]\right] } & =-\left[e_{2},\left[e_{6}, e_{1}\right]\right]-\left[e_{6},\left[e_{1}, e_{2}\right]\right] \\
& =\left[e_{2}, e_{4}\right]+\alpha_{22}\left[e_{2}, e_{6}\right]+\alpha_{23}\left[e_{3}, e_{6}\right]+\alpha_{24}\left[e_{4}, e_{6}\right] \\
{\left[e_{1},\left[e_{4}, e_{3}\right]\right] } & =-\left[e_{4},\left[e_{3}, e_{1}\right]\right]-\left[e_{3},\left[e_{1}, e_{4}\right]\right] \\
& =\left[e_{4}, e_{2}\right]+\alpha_{44}\left[e_{4}, e_{3}\right]+\alpha_{45}\left[e_{5}, e_{3}\right]+\alpha_{46}\left[e_{6}, e_{3}\right] \\
{\left[e_{1},\left[e_{3}, e_{5}\right]\right] } & =-\left[e_{3},\left[e_{5}, e_{1}\right]\right]-\left[e_{5},\left[e_{1}, e_{3}\right]\right]=\left[e_{2}, e_{5}\right],
\end{aligned}
$$

which shows that $\left[e_{2}, e_{4}\right] \in S$ (provided that the characteristic of $F$ is not 3). Finally,

$$
\begin{aligned}
{\left[e_{1},\left[e_{2}, e_{5}\right]\right] } & =-\left[e_{2},\left[e_{5}, e_{1}\right]\right]-\left[e_{5},\left[e_{1}, e_{2}\right]\right. \\
& =\left[e_{2}, e_{3}\right]+\alpha_{22}\left[e_{2}, e_{5}\right]+\alpha_{23}\left[e_{3}, e_{5}\right]+\alpha_{24}\left[e_{4}, e_{5}\right]
\end{aligned}
$$

from which we have that $\left[e_{2}, e_{3}\right] \in S$. As $A$ is an ideal of $N$ and $(A+$ $\left.\left[A, e_{1}\right]\right)^{2} \subseteq A, N^{2}$ is contained in $A$ and hence in $Z(N)$. It follows that $N^{3}=0$ and $N^{2}$ has dimension at most five.

Case B1 Suppose that $e_{7}, e_{8} \in Z(N)$. Then $4 \geq \operatorname{dim}([L, N]+Z(N)) \geq 3$, since $e_{2}, e_{3}, e_{4} \in[L, N]+Z(N)$.

(i) Suppose that $\operatorname{dim}([L, N]+Z(N))=3$, so $\alpha_{25}=\alpha_{26}=\alpha_{45}=\alpha_{46}=0$. Then $F\left[e_{1}, e_{2}\right] \subseteq\left[L,\left[L,\left[L, A^{L}\right]\right]\right]+Z(N)$ which has dimension $\operatorname{dim} Z(N)+1$.

Suppose further that $\left[e_{1}, e_{2}\right]=0$, so $\alpha_{22}=\alpha_{23}=\alpha_{24}=0$ and $F e_{2}+Z(N)$ is an abelian ideal of dimension $\operatorname{dim} N-4$. Then

$$
\begin{aligned}
{\left[e_{1},\left[e_{2}, e_{3}\right]\right] } & =-\left[e_{2},\left[e_{3}, e_{1}\right]\right]-\left[e_{3},\left[e_{1}, e_{2}\right]\right]=0 \text { and } \\
{\left[e_{1},\left[e_{2}, e_{4}\right]\right] } & =-\left[e_{2},\left[e_{4}, e_{1}\right]\right]-\left[e_{4},\left[e_{1}, e_{2}\right]\right. \\
& =\alpha_{43}\left[e_{2}, e_{3}\right]+\alpha_{44}\left[e_{2}, e_{4}\right]
\end{aligned}
$$

Hence, $F\left[e_{2}, e_{3}\right]+F\left[e_{2}, e_{4}\right]$ is an ideal of $L$. It has dimension at most two, so

$$
0=\left[e_{1},\left[e_{1},\left[e_{2}, e_{4}\right]\right]\right]=\alpha_{43} \alpha_{44}\left[e_{2}, e_{3}\right]+\alpha_{44}^{2}\left[e_{2}, e_{4}\right] .
$$


If $\alpha_{44} \neq 0$ we have $\left[e_{2}, \alpha_{43} e_{3}+\alpha_{44} e_{4}\right]=0$. It follows that $\alpha_{43} e_{3}+\alpha_{44} e_{4} \in$ $C_{L}\left(F e_{2}+Z(N)\right)$ and $L$ has an abelian ideal of dimension $\operatorname{dim} N-3$. If $\alpha_{44}=$ 0 , equations (38)-(41) give $\left[e_{2}, e_{6}\right]=2\left[e_{4}, e_{3}\right]+\alpha_{43}\left[e_{3}, e_{5}\right]$ and $3\left[e_{2}, e_{4}\right]=$ $\alpha_{43}\left[e_{2}, e_{5}\right]$, whence $\left[e_{2}, 3 e_{4}-\alpha_{43} e_{5}\right]=0$. Then $3 e_{4}-\alpha_{43} e_{5} \in C_{L}\left(F e_{2}+Z(N)\right)$ and again $L$ has an abelian ideal of dimension $\operatorname{dim} N-3$.

So suppose now that $\left[e_{1}, e_{2}\right] \neq 0$. Then $F\left[e_{1}, e_{2}\right]+Z(N)=\left[L,\left[L,\left[L, A^{L}\right]\right]\right]+$ $Z(N)$. Hence

$\left[e_{1},\left[e_{1}, e_{2}\right]\right]=\alpha_{22}\left(\alpha_{22} e_{2}+\alpha_{23} e_{3}+\alpha_{24} e_{4}\right)+\alpha_{23} e_{2}+\alpha_{24}\left(\alpha_{43} e_{3}+\alpha_{44} e_{4}\right)+z$,

where $z \in Z(N)$. Since this must belong to $Z(N)$ we have

$$
\begin{aligned}
\alpha_{22}^{2}+\alpha_{23} & =0 \\
\alpha_{22} \alpha_{23}+\alpha_{24} \alpha_{43} & =0 \\
\alpha_{22} \alpha_{24}+\alpha_{24} \alpha_{44} & =0
\end{aligned}
$$

These equations yield that $\left[e_{1}, e_{2}\right]=\alpha_{22} e_{2}-\alpha_{22}^{2} e_{3}+\alpha_{24} e_{4}$ where

$$
\alpha_{22}=-\alpha_{44} \text { and } \alpha_{24} \alpha_{43}=\alpha_{22}^{3}
$$

since $\alpha_{24}=0$ implies that $\alpha_{22}=\alpha_{23}=0$ and $\left[e_{1}, e_{2}\right]=0$. Suppose first that $\alpha_{43}=\alpha_{44}=0$, so $F e_{4}+Z(N)$ is an abelian ideal of dimension $\operatorname{dim} N-4$. Then (38)-(41) give $\left[e_{2}, e_{6}\right]=2\left[e_{4}, e_{3}\right]$ and $3\left[e_{2}, e_{4}\right]=0$ and again $L$ has an abelian ideal of dimension $\operatorname{dim} N-3$. So suppose that at least one of $\alpha_{43}$ and $\alpha_{44}$ is non-zero. If any of $\alpha_{22}, \alpha_{23}$ or $\alpha_{24}$ is zero then they are all zero, so we can assume that they are all non-zero.

Now

$$
\begin{aligned}
{\left[e_{1},\left[e_{2}, e_{3}\right]\right] } & =-\left[e_{2},\left[e_{3}, e_{1}\right]\right]-\left[e_{3},\left[e_{1}, e_{2}\right]\right] \\
& =\alpha_{22}\left[e_{2}, e_{3}\right]+\alpha_{24}\left[e_{2}, e_{4}\right] \\
{\left[e_{1},\left[e_{2}, e_{4}\right]\right] } & =-\left[e_{2},\left[e_{4}, e_{1}\right]\right]-\left[e_{4},\left[e_{1}, e_{2}\right]\right] \\
& =\alpha_{43}\left[e_{2}, e_{3}\right]+\alpha_{44}\left[e_{2}, e_{4}\right]+\alpha_{22}\left[e_{2}, e_{4}\right]+\alpha_{23}\left[e_{3}, e_{4}\right] \\
& =\alpha_{43}\left[e_{2}, e_{3}\right]+\alpha_{23}\left[e_{3}, e_{4}\right] \text { by (iv) } \\
{\left[e_{1},\left[e_{3}, e_{4}\right]\right] } & =\left[e_{2}, e_{4}\right]-\alpha_{22}\left[e_{3}, e_{4}\right] \text { by (40) and (iv) }
\end{aligned}
$$

It follows that $K=F\left[e_{2}, e_{3}\right]+F\left[e_{2}, e_{4}\right]+F\left[e_{3}, e_{4}\right]$ is an ideal of $L$ of dimension at most three. Thus $\left[e_{1},\left[e_{1},\left[e_{1},\left[e_{2}, e_{3}\right]\right]\right]\right]=0$. Now

$$
\begin{aligned}
{\left[e_{1},\left[e_{1},\left[e_{2}, e_{3}\right]\right]\right] } & =\alpha_{22}^{2}\left[e_{2}, e_{3}\right]+\alpha_{22} \alpha_{24}\left[e_{2}, e_{4}\right]+\alpha_{24} \alpha_{43}\left[e_{2}, e_{3}\right]+\alpha_{24} \alpha_{23}\left[e_{3}, e_{4}\right] \\
& =\alpha_{22}^{2}\left(1+\alpha_{22}\right)\left[e_{2}, e_{3}\right]+\alpha_{22} \alpha_{24}\left[e_{2}, e_{4}\right]+\alpha_{24} \alpha_{23}\left[e_{3}, e_{4}\right] \text { by (iv) }
\end{aligned}
$$


whence

$$
\begin{aligned}
0 & =\alpha_{22}^{3}\left(1+\alpha_{22}\right)\left[e_{2}, e_{3}\right]+\alpha_{22}^{2} \alpha_{24}\left(1+\alpha_{22}\right)\left[e_{2}, e_{4}\right]+\alpha_{22} \alpha_{24} \alpha_{43}\left[e_{2}, e_{3}\right] \\
& +\alpha_{22} \alpha_{23} \alpha_{24}\left[e_{3}, e_{4}\right]+\alpha_{24} \alpha_{23}\left[e_{2}, e_{4}\right]-\alpha_{22} \alpha_{23} \alpha_{24}\left[e_{3}, e_{4}\right] \\
& =\alpha_{22}^{3}\left(1+2 \alpha_{22}\right)\left[e_{2}, e_{3}\right]+\alpha_{24} \alpha_{22}^{3}\left[e_{2}, e_{4}\right] \text { by (i) }
\end{aligned}
$$

This implies that $\left[e_{2}, e_{3}\right]$ and $\left[e_{2}, e_{4}\right]$ are linearly dependent and $K$ has dimension at most two. But then $\left[e_{1},\left[e_{1},\left[e_{2}, e_{3}\right]\right]\right]=0$ which shows that all three are linearly dependent. It follows that $\operatorname{dim} N^{2} \leq 3$. Hence

$$
\lambda_{1}\left[e_{3},\left[e_{1}, e_{2}\right]\right]+\lambda_{2}\left[e_{4},\left[e_{1}, e_{2}\right]\right]+\lambda_{3}\left[e_{5},\left[e_{1}, e_{2}\right]\right]+\lambda_{4}\left[e_{6},\left[e_{1}, e_{2}\right]\right]=0
$$

for some $\lambda_{i} \in F$, not all zero $(i=1,2,3,4)$. Thus,

$$
\lambda_{1} e_{3}+\lambda_{2} e_{4}+\lambda_{3} e_{5}+\lambda_{4} e_{6} \in C_{L}\left(F\left[e_{1}, e_{2}\right]+Z(N)\right)
$$

and $L$ has an abelian ideal of dimension $\operatorname{dim} N-3$.

(ii) Suppose now that $\operatorname{dim}([L, N]+Z(N))=4$. Then we proceed exactly as in Case A1(ii).

Case B2 If $\alpha_{75}=\alpha_{76}=\alpha_{86}=0$ we are in case B1, so we now consider the situation when at least one of these is non-zero.

(i) Suppose that $\alpha_{86}=0$, so $e_{8} \in Z(N)$ and $\operatorname{dim} Z(N) \leq \operatorname{dim} N-6$. Then

$$
\begin{aligned}
0 & =\left[e_{1},\left[e_{4}, e_{7}\right]\right]=-\left[e_{4},\left[e_{7}, e_{1}\right]\right]-\left[e_{7},\left[e_{1}, e_{4}\right]\right] \\
& =\alpha_{75}\left[e_{4}, e_{5}\right]+\alpha_{76}\left[e_{4}, e_{6}\right]
\end{aligned}
$$

Also, multiplying (46) by $e_{1}$ and using (37) gives

$$
\begin{aligned}
0 & =\alpha_{75}\left[e_{4}, e_{3}\right]+\alpha_{75} \alpha_{44}\left[e_{4}, e_{5}\right]+\alpha_{75} \alpha_{43}\left[e_{3}, e_{5}\right]+\alpha_{76} \alpha_{43}\left[e_{3}, e_{6}\right] \\
& +\alpha_{76} \alpha_{44}\left[e_{4}, e_{6}\right] \\
& =\alpha_{75}\left[e_{4}, e_{3}\right]+\alpha_{76} \alpha_{43}\left[e_{4}, e_{5}\right]+\alpha_{75} \alpha_{43}\left[e_{3}, e_{5}\right] \text { using }(32)
\end{aligned}
$$

If $\alpha_{75} \neq 0$ equations (46) and (47) imply that $\operatorname{dim} N^{2} \leq 3$. Now there exists an abelian ideal $B=F b+Z(N) \subseteq N$. If this is a maximal abelian ideal then, by Lemma 3.1, there exist $\lambda_{i} \in F$, not all zero, such that $B=\sum_{i=4}^{7} \lambda_{i} e_{i}+A_{L}$. But then $e_{7} \in C_{L}(B)=B$ and we are in case B1. Thus, there is an abelian ideal $C=F c+B \subseteq N$. Suppose that this is a maximal abelian ideal of $L$. As above there exist $\mu_{i} \in F$, not all zero, such that $\sum_{i=4}^{7} \mu_{i} e_{i} \in C_{L}(F c+Z(N))$. It follows that $e_{7} \in C_{L}(C)=C$ and we are in case B1 again. If this is not maximal then there is an abelian ideal of dimension $\operatorname{dim} N-3$. 
So suppose that $\alpha_{75}=0$. Then $\alpha_{76} \neq 0$ and (46) implies that $\left[e_{4}, e_{6}\right]=0$ giving $\operatorname{dim} N^{2} \leq 4$. Now we can argue as in the previous paragraph using $\sum_{i=3}^{7} \lambda_{i} e_{i}$ and $\sum_{i=3}^{7} \mu_{i} e_{i}$.

(ii) Suppose that $\alpha_{86} \neq 0$. Then

$$
0=\left[e_{1},\left[e_{4}, e_{8}\right]\right]=-\left[e_{4},\left[e_{8}, e_{1}\right]\right]-\left[e_{8},\left[e_{1}, e_{4}\right]\right]=\alpha_{86}\left[e_{4}, e_{6}\right]
$$

so $\left[e_{4}, e_{6}\right]=0$ and $\operatorname{dim} N^{2} \leq 4$. Moreover, (46) implies that $\alpha_{75}=0$ or $\left[e_{4}, e_{5}\right]=0$. The latter yields that $\left[e_{2}, e_{8}\right]=0$ (using (32)) and we are in Case B2(i). If $\alpha_{75}=0$ then $\left[e_{2}, \alpha_{76} e_{8}-\alpha_{86} e_{7}\right]=0$, so, replacing $e_{8}$ by $\alpha_{76} e_{8}-\alpha_{86} e_{7}$ we can assume that $\left[e_{2}, e_{8}\right]=0$ and we are in Case B2(i) again.

Case C: Suppose that $N=F e_{2}+F e_{3}+F e_{4}+A$ where $e_{4}=\left[e_{1}, e_{7}\right]$, $e_{3}=\left[e_{1}, e_{6}\right], e_{2}=\left[e_{1}, e_{5}\right]$. In particular, this is the case if $N=A+$ $\left[A, e_{1}\right]$. Replacing $e_{j}$ by $e_{j}-\alpha_{j 2} e_{5}-\alpha_{j 3} e_{6}-\alpha_{j 4} e_{7}$ we can assume that $\left[e_{1}, e_{j}\right]=\sum_{i=5}^{n} \alpha_{j i} e_{i}$ for $j \geq 8$. Then, as before, $F e_{8}+\ldots+F e_{n} \subseteq Z(N)$ and $\operatorname{dim} Z(N) \geq \operatorname{dim} N-6$. Now

$$
\begin{aligned}
0=\left[e_{1},\left[e_{5}, e_{6}\right]\right] & =-\left[e_{5},\left[e_{6}, e_{1}\right]\right]-\left[e_{6},\left[e_{1}, e_{5}\right]\right] \\
& =\left[e_{5}, e_{3}\right]+\left[e_{2}, e_{6}\right] \\
0=\left[e_{1},\left[e_{5}, e_{7}\right]\right] & =-\left[e_{5},\left[e_{7}, e_{1}\right]\right]-\left[e_{7},\left[e_{1}, e_{5}\right]\right] \\
& =\left[e_{5}, e_{4}\right]+\left[e_{2}, e_{7}\right] \\
0=\left[e_{1},\left[e_{6}, e_{7}\right]\right] & =-\left[e_{6},\left[e_{7}, e_{1}\right]\right]-\left[e_{7},\left[e_{1}, e_{6}\right]\right] \\
& =\left[e_{6}, e_{4}\right]+\left[e_{3}, e_{7}\right]
\end{aligned}
$$

If $\alpha_{23} \neq 0$ we can replace $e_{3}$ by $\left[e_{1}, e_{2}\right]$ and we are in Case $\mathrm{B}$, so we can assume that $\alpha_{23}=0$. Likewise, if $\alpha_{24} \neq 0$ we can replace $e_{4}$ by $\left[e_{1}, e_{2}\right]$, so we can assume that $\alpha_{24}=0$. Similarly, we can assume that

$$
\alpha_{32}=\alpha_{34}=\alpha_{42}=\alpha_{43}=0 .
$$


Then, multiplying each of (49)-(51) by $e_{1}$ gives

$$
\begin{aligned}
{\left[e_{1},\left[e_{2}, e_{6}\right]\right] } & =-\left[e_{2},\left[e_{6}, e_{1}\right]\right]-\left[e_{6},\left[e_{1}, e_{2}\right]\right] \\
& =\left[e_{2}, e_{3}\right]+\alpha_{22}\left[e_{2}, e_{6}\right] \\
{\left[e_{1},\left[e_{3}, e_{5}\right]\right] } & =-\left[e_{3},\left[e_{5}, e_{1}\right]\right]-\left[e_{5},\left[e_{1}, e_{3}\right]\right] \\
& =\left[e_{3}, e_{2}\right]+\alpha_{33}\left[e_{3}, e_{5}\right] \\
{\left[e_{1},\left[e_{2}, e_{7}\right]\right] } & =-\left[e_{2},\left[e_{7}, e_{1}\right]\right]-\left[e_{7},\left[e_{1}, e_{2}\right]\right] \\
& =\left[e_{2}, e_{4}\right]+\alpha_{22}\left[e_{2}, e_{7}\right] \\
{\left[e_{1},\left[e_{4}, e_{5}\right]\right] } & =-\left[e_{4},\left[e_{5}, e_{1}\right]\right]-\left[e_{5},\left[e_{1}, e_{4}\right]\right] \\
& =\left[e_{4}, e_{2}\right]+\alpha_{44}\left[e_{4}, e_{5}\right] \\
{\left[e_{1},\left[e_{3}, e_{7}\right]\right] } & =-\left[e_{3},\left[e_{7}, e_{1}\right]\right]-\left[e_{7},\left[e_{1}, e_{3}\right]\right] \\
& =\left[e_{3}, e_{4}\right]+\alpha_{33}\left[e_{3}, e_{7}\right] \\
{\left[e_{1},\left[e_{4}, e_{6}\right]\right] } & =-\left[e_{4},\left[e_{6}, e_{1}\right]\right]-\left[e_{6},\left[e_{1}, e_{4}\right]\right] \\
& =\left[e_{4}, e_{3}\right]+\alpha_{44}\left[e_{4}, e_{6}\right]
\end{aligned}
$$

Equations (52)-(57) yield

$$
\begin{aligned}
& 2\left[e_{2}, e_{3}\right]=\left(\alpha_{33}-\alpha_{22}\right)\left[e_{2}, e_{6}\right] \\
& 2\left[e_{2}, e_{4}\right]=\left(\alpha_{44}-\alpha_{22}\right)\left[e_{2}, e_{7}\right] \\
& 2\left[e_{3}, e_{4}\right]=\left(\alpha_{44}-\alpha_{33}\right)\left[e_{3}, e_{7}\right]
\end{aligned}
$$

Substituting (58) back into (52) gives

$$
\left[e_{1},\left[e_{2}, e_{6}\right]\right]=\frac{1}{2}\left(\alpha_{22}+\alpha_{33}\right)\left[e_{2}, e_{6}\right]
$$

whence

$$
\left(\operatorname{ad} e_{1}\right)^{r}\left(\left[e_{2}, e_{6}\right]\right)=\frac{1}{2^{r}}\left(\alpha_{22}+\alpha_{33}\right)\left[e_{2}, e_{6}\right]
$$

and nilpotency gives

$$
\left[e_{2}, e_{6}\right]=\left[e_{2}, e_{3}\right]=0 \quad \text { or } \quad \alpha_{22}=-\alpha_{33} \text { and }\left[e_{2}, e_{3}\right]=\alpha_{33}\left[e_{2}, e_{6}\right]
$$

Similarly,

$$
\begin{array}{ll}
{\left[e_{2}, e_{7}\right]=\left[e_{2}, e_{4}\right]=0 \quad \text { or }} & \alpha_{22}=-\alpha_{44} \text { and }\left[e_{2}, e_{4}\right]=\alpha_{44}\left[e_{2}, e_{7}\right] \\
{\left[e_{3}, e_{7}\right]=\left[e_{3}, e_{4}\right]=0 \quad \text { or } \quad \alpha_{33}=-\alpha_{44} \text { and }\left[e_{3}, e_{4}\right]=\alpha_{44}\left[e_{3}, e_{7}\right]}
\end{array}
$$

(i) Suppose that $\operatorname{dim}([L, N]+Z(N))=3$, so

$$
\alpha_{25}=\alpha_{26}=\alpha_{27}=\alpha_{35}=\alpha_{36}=\alpha_{37}=\alpha_{45}=\alpha_{46}=\alpha_{47}=0
$$


. Then $\left[e_{1}, e_{2}\right]=\alpha_{22} e_{2}$ and nilpotency requires that $\alpha_{22}=0$. Similarly, $\alpha_{33}=\alpha_{44}=0$ and (58),(59),(60) imply that $F e_{2}+F e_{3}+F e_{4}+Z(N)$ is an abelian ideal of dimension $\operatorname{dim} N-3$.

(ii) So suppose now that $\operatorname{dim}([L, N]+Z(N))=4$, so $[L, A]+Z(N)=$ $F e_{2}+F e_{3}+F e_{4}+F a+Z(N)$, where $a \in A$. Then $\left[e_{1}, a\right]=\beta_{1} a+\beta_{2} e_{2}+$ $\beta_{3} e_{3}+\beta_{4} e_{4}+z$, where $\beta_{i} \in F(1 \leq i \leq 4)$ and $z \in Z(N)$. We must have at least one of $\beta_{2}, \beta_{3}, \beta_{4}$ non-zero, by nilpotency. If $\beta_{i} \neq 0$ for $2 \leq i \leq 4$, by replacing $e_{i}$ by $\left[e_{1}, a\right]$ we can assume that $a=e_{i+3}$. By symmetry we can suppose that $a=e_{5}$.

Then $F e_{2}+F e_{5}+Z(N)$ is an ideal of $L$. Hence nilpotency gives that $\left[e_{1}, e_{2}\right]=\left[e_{1},\left[e_{1}, e_{5}\right]\right] \in Z(N)$. We then have that $F e_{2}+F e_{3}+F e_{5}+Z(N)$ is an ideal of $L$, whence

$$
\left(\operatorname{ad} e_{1}\right)^{3}\left(e_{3}\right)=\alpha_{33} \alpha_{35} e_{2}+\alpha_{33}^{3} e_{3}+\alpha_{33}^{2} \alpha_{35} e_{5}+z \in Z(N),
$$

where $z \in Z(N)$. This yields that $\alpha_{33}=0$. Similarly, $\alpha_{44}=0$, so (58), (59, (60) give $\left[e_{2}, e_{3}\right]=\left[e_{2}, e_{4}\right]=\left[e_{3}, e_{4}\right]=0$.

If $\alpha_{35}=0$ then $B=F e_{2}+F e_{3}+Z(N)$ is an abelian ideal of $L$ and is not maximal, since $e_{4} \in C_{L}(B)$. Similarly if $\alpha_{45}=0$. If neither is zero, then $B=F e_{2}+F\left(\alpha_{45} e_{3}-\alpha_{35} e_{4}\right)+Z(N)$ is an abelian ideal of $L$ and, again, is not maximal, since $e_{3}, e_{4} \in C_{L}(B)$.

(iii) Finally suppose that $\operatorname{dim}([L, N]+Z(N))=5$, so $[L, N]+Z(N)=$ $F e_{2}+F e_{3}+F e_{4}+F a_{1}+F a_{2}+Z(N)$ for some $a_{1}, a_{2} \in F e_{5}+F e_{6}+F e_{7}$. If $\left[e_{1}, a_{1}\right]$ and $\left[e_{1}, a_{2}\right]$ are linearly dependent, there exists a non-trivial linear combination $x=\sum_{i=5}^{7} \lambda_{i} e_{i}$ such that $\left[e_{1}, x\right]=0$. But this implies that $e_{2}, e_{3}, e_{4}$ are linearly dependent. So, by symmetry we assume that $a_{1}=e_{5}$ and $a_{2}=e_{6}$. Hence $K=\sum_{i=2}^{6} F e_{i}+Z(N)$ is an ideal of $L$.

We have that $\left[e_{1}, e_{4}\right] \in K$, so let $\left[e_{1}, e_{4}\right]=\sum_{i=4}^{6} \alpha_{4 i} e_{i}+z(z \in Z(N))$. The coefficient of $e_{4}$ in $\left(\operatorname{ad} e_{1}\right)^{k}\left(e_{4}\right)$ is $\alpha_{44}^{k}$ and nilpotency gives that $\alpha_{44}=0$, so $\left[e_{1}, e_{4}\right] \in A$. Moreover, (62) and (63) imply that $\left[e_{2}, e_{4}\right]=\left[e_{3}, e_{4}\right]=0$. Now $e_{1}, e_{7}$ and $Z(N)$ generate $L$. Put $e_{7}=n_{1}$. Then $L=e_{1}+\sum_{i=1}^{6} n_{i}+$ $Z(N)$, where $\left[e_{1}, n_{i}\right]=n_{i+1}$ for $1 \leq i \leq 5$ and $\left[e_{1}, n_{6}\right] \in Z(N)$. Then $n_{3}=\left[e_{1}, e_{4}\right] \in A \cap K=\alpha_{45} e_{5}+\alpha_{46} e_{6}, n_{4}=\alpha_{45} e_{2}+\alpha_{46} e_{3}$, so

$$
\left[n_{1}, n_{3}\right]=0 \quad \text { and } \quad\left[n_{2}, n_{4}\right]=0 .
$$


Hence

$$
\begin{aligned}
& {\left[e_{1},\left[n_{1}, n_{3}\right]\right]=-\left[n_{1},\left[n_{3}, e_{1}\right]\right]-\left[n_{3},\left[e_{1}, n_{1}\right]\right]=\left[n_{1}, n_{4}\right]+\left[n_{2}, n_{3}\right]=0} \\
& {\left[e_{1},\left[n_{1}, n_{4}\right]\right]=-\left[n_{1},\left[n_{4}, e_{1}\right]\right]-\left[n_{4},\left[e_{1}, n_{1}\right]\right]=\left[n_{1}, n_{5}\right] \text { and }} \\
& {\left[e_{1},\left[n_{2}, n_{3}\right]\right]=-\left[n_{2},\left[n_{3}, e_{1}\right]\right]-\left[n_{3},\left[e_{1}, n_{2}\right]\right]=0 \text { so }} \\
& {\left[n_{1}, n_{5}\right]=0} \\
& {\left[e_{1},\left[n_{1}, n_{5}\right]\right]=-\left[n_{1},\left[n_{5}, e_{1}\right]\right]-\left[n_{5},\left[e_{1}, n_{1}\right]\right]=\left[n_{1}, n_{6}\right]+\left[n_{2}, n_{5}\right]=0} \\
& {\left[e_{1},\left[n_{1}, n_{6}\right]\right]=-\left[n_{1},\left[n_{6}, e_{1}\right]\right]-\left[n_{6},\left[e_{1}, n_{1}\right]\right]=\left[n_{2}, n_{6}\right] \text { and }} \\
& {\left[e_{1},\left[n_{2}, n_{5}\right]\right]=-\left[n_{2},\left[n_{5}, e_{1}\right]\right]-\left[n_{5},\left[e_{1}, n_{2}\right]\right]=\left[n_{2}, n_{6}\right]+\left[n_{3}, n_{5}\right] \text { so }} \\
& 2\left[n_{2}, n_{6}\right]+\left[n_{3}, n_{5}\right]=0 \\
& {\left[e_{1},\left[n_{2}, n_{6}\right]\right]=-\left[n_{2},\left[n_{6}, e_{1}\right]\right]-\left[n_{6},\left[e_{1}, n_{2}\right]\right]=\left[n_{3}, n_{6}\right] \text { and }} \\
& {\left[e_{1},\left[n_{3}, n_{5}\right]\right]=-\left[n_{3},\left[n_{5}, e_{1}\right]\right]-\left[n_{5},\left[e_{1}, n_{3}\right]\right]=\left[n_{3}, n_{6}\right]+\left[n_{4}, n_{5}\right] \text { so }} \\
& 3\left[n_{3}, n_{6}\right]+\left[n_{4}, n_{5}\right]=0 \\
& {\left[e_{1},\left[n_{3}, n_{6}\right]\right]=-\left[n_{3},\left[n_{6}, e_{1}\right]\right]-\left[n_{6},\left[e_{1}, n_{3}\right]\right]=\left[n_{4}, n_{6}\right] \text { and }} \\
& {\left[e_{1},\left[n_{4}, n_{5}\right]\right]=-\left[n_{4},\left[n_{5}, e_{1}\right]\right]-\left[n_{5},\left[e_{1}, n_{4}\right]\right]=\left[n_{4}, n_{6}\right] \text { so }} \\
& 4\left[n_{4}, n_{6}\right]=0 \\
& {\left[e_{1},\left[n_{4}, n_{6}\right]\right]=-\left[n_{4},\left[n_{6}, e_{1}\right]\right]-\left[n_{6},\left[e_{1}, n_{4}\right]\right]=\left[n_{5}, n_{6}\right]=0}
\end{aligned}
$$

Also

$$
\begin{gathered}
{\left[e_{1},\left[n_{2}, n_{4}\right]\right]=-\left[n_{2},\left[n_{4}, e_{1}\right]\right]-\left[n_{4},\left[e_{1}, n_{2}\right]\right]=\left[n_{2}, n_{5}\right]+\left[n_{3}, n_{4}\right]=0} \\
{\left[e_{1},\left[n_{3}, n_{4}\right]\right]=-\left[n_{3},\left[n_{4}, e_{1}\right]\right]-\left[n_{4},\left[e_{1}, n_{3}\right]\right]=\left[n_{3}, n_{5}\right] \text { so }} \\
{\left[n_{2}, n_{6}\right]+2\left[n_{3}, n_{5}\right]=0}
\end{gathered}
$$

Then (67) and (73) imply that $\left[n_{2}, n_{6}\right]=\left[n_{3}, n_{5}\right]=0$. But now (68) gives $\left[n_{3}, n_{6}\right]=0$ and $(69)$ then yields that $\left[n_{4}, n_{5}\right]=0$. Together with (70) and (71) this shows that $F n_{4}+F n_{5}+F n_{6}+Z(N)$ is an abelian ideal of dimension $\operatorname{dim} N-3$, completing the proof.

This, of course, leaves open the question of whether the restrictions on the characteristic are necessary: 2 needs to be excluded as shown in [5], and probably 3 , but the author is unsure of 5 . Also whether the above result holds for supersolvable algebras, and whether these results hold more generally. However, the equations become increasingly complex and so more powerful methods may be required to tackle these questions. 


\section{References}

[1] D.W. Barnes and M.L. Newell, 'Some theorems on saturated homomorphs of soluble Lie algebras', Math. Zeit. 115 (1970), 179-187.

[2] D. Burde, C. Steinhoff, 'Classification of orbit closures of 4dimensional complex Lie algebras', J. Algebra 214(2) (1999), 729-739.

[3] D. Burde and M. Ceballos, 'Abelian ideals of maximal dimension for solvable Lie algebras', J. Lie Theory 22(3) (2012), 741-756.

[4] , M. Ceballos, 'Abelian subalgebras and ideals of maximal dimension in Lie algebras', Thesis, University of Seville (2012), https://core.ac.uk/reader/51399078.

[5] M. Ceballos And D.A. Towers, 'On abelian subalgebras and ideals of maximal dimension in supersolvable Lie algebras', J. Pure Appl. Algebra 218 (2014), 497-503.

[6] V. V. Gorbatsevich, 'On the level of some solvable Lie algebras', Siberian Math. J. 39 (5) (1998), 872-883.

[7] F. Grunewald, J. O'Halloran, 'Varieties of nilpotent Lie algebras of dimension less than six', J. Algebra 112 (2) (1988), 315-325.

[8] N. Jacobson, 'Schur's Theorems on Commutative Matrices', Bull. Amer. Math. Soc. 50 (1944), 431-436.

[9] N. Jacobson, 'Lie algebras', New York: Dover Publ. (1979).

[10] B. Kostant, 'The set of abelian ideals of a Borel subalgebra, Cartan decompositions, and discrete series representations', Internat. Math. Res. Notices no. 5, (1998), 225-252.

[11] B. Kostant, 'Powers of the Euler product and commutative subalgebras of a complex simple Lie algebra', Invent. Math. 158 (2004), 181-226.

[12] D. V. Maksimenko, 'On action of outer derivations on nilpotent ideals of Lie algebras', Algebra Discrete Math. 1 (2009), 74-82.

[13] A. Malcev, 'Commutative subalgebras of semi-simple Lie algebras', Amer. Math. Soc. Transl. 1951(40) (1951), 15 pp.

[14] M. Nesterenko, R. Popovych, 'Contractions of low-dimensional Lie algebras', J. Math. Phys. 47 (12) (2006), 123515, 45 pp. 
[15] A.I.Ooms, 'The maximaql abelian dimension of a Lie algebra, Rentschler's property and Milovanov's conjecture', Algebras and Rep. Thry. 23 (2020), 963-999.

[16] I. Schur, 'Zur Theorie vertauschbarer Matrizen', J. Reine. Angew. Math, 130 (1905), 66-76.

[17] C. Seeley, 'Degenerations of 6-dimensional nilpotent Lie algebras over $\mathbb{C}^{\prime}$, Comm. in Algebra 18 (1990), 3493-3505.

[18] R. Suter, 'Abelian ideals in a Borel subalgebra of a complex simple Lie algebra', Invent. Math. 156 (2004), 175-221.

[19] D. A. Towers, 'A Frattini Theory for Algebras', Proc. London Math. Soc. (3) 27 (1973), 440-462.

[20] D. A. Towers, 'On certain decompositions of solvable Lie algebras', J. Lie Theory 24 (4) (2014), 969-978. 